\title{
A NOTE ON INTERPOLATION
}

\section{BOAZ NATZITZ}

The purpose of this note is to give a direct proof of the following theorem: [2].

Let $A$ be a uniform algebra on a compact Hausdorff space $X$ and $E$ a closed subset of $X$. If $\operatorname{Re} A \mid E=C_{R}(E)$, then $A \mid E=C(E)$.

Proof. By a result of Glicksberg [1] we have $A \mid E=C(E)$ if and only if there is a constant $c$ such that $\left\|\mu_{E}\right\| \leqq c\left\|\mu_{E},\right\|$ whenever $\mu$ is a complex Borel measure on $X$ orthogonal to $A$ and $\mu_{E}, \mu_{E}$, are the restriction of $\mu$ to $E$ and $E^{\prime}$ (the complement of $E$ ) respectively. Now let $\mu$ be a measure orthogonal to $A$. Choose $f \in C_{R}(E), 0 \leqq f \leqq 2 \pi$, such that $\int_{E} e^{i f} d \mu$ is $\epsilon$-close to $\left\|\mu_{E}\right\|$. By applying the open mapping theorem to $A \rightarrow \operatorname{Re} A \mid E=C_{R}(E)$ one sees that there exists a constant $c_{1}$ such that for every $g \in C_{R}(E)$ there is a $\phi \in A, \operatorname{Re} \phi \mid E=g$, and $\|\phi\| \leqq c_{1}\|g\|$. In particular if $\operatorname{Re} \phi \mid E=f$ (as above) we have that

$$
\int_{E} e^{i \phi} d \mu \text { is close to } c_{2}\left\|\mu_{E}\right\| \text { where } \exp \left(-2 \pi c_{1}\right) \leqq c_{2} \leqq \exp \left(2 \pi c_{1}\right) \text {. }
$$

Indeed if $\phi=\tilde{f}+i g, \tilde{f}$ a real extension of $f$ to $X$ then $\left|\int_{E} e^{i f-g} d \mu\right|$ $\geqq\left[\left\|\mu_{E}\right\|-\epsilon\right] \exp \left(-2 \pi c_{1}\right)$. On the other hand since $e^{i \phi} \in A$ we have that

$$
\int_{E} e^{i \phi} d \mu=-\int_{E^{\prime}} e^{i \phi} d \mu \text {, and }\left|\int_{E^{\prime}} e^{i \phi} d \mu\right| \leqq \exp \left(2 \pi c_{1}\right)\left\|\mu_{E^{\prime}}\right\| \text {. }
$$

Combining the above results we get

$$
\left\|\mu_{E}\right\|-\epsilon \leqq \exp \left(4 \pi c_{1}\right)\left\|\mu_{E^{\prime}}\right\| \text { as required. }
$$

1. I. Glicksberg, Measures orthogonal to algebras and sets of antisymmetry, Trans. Amer. Math. Soc. 105 (1962), 415-435. MR 30 \#4164.

2. S. J. Sidney and E. L. Stout, $A$ note on interpolation, Proc. Amer. Math. Soc. 19 (1968), 380-382. MR 36 \#6944.

McGill University, Montreal, Canada

Received by the editors May 24, 1969. 